\title{
Trois congrégations religieuses enseignantes au mont Saint-Bruno : \\ 2. Les pères Trinitaires \\ 3. Les religieuses des Sacrés-Cœurs et de l'adoration perpétuelle
}

\section{Andrée Dufour}

Dans un texte précédent, ${ }^{1}$ nous avons fait état de la présence éducative de la congrégation des frères de Saint-Gabriel au Mont Saint-Bruno. Il sera ici question des deux autres communautés religieuses enseignantes qui se sont installées sur les flancs de cette colline montérégienne et qui y ont fait œuvre éducative : l'Ordre des Trinitaires et les religieuses des Sacrés-Cours et de l'adoration perpétuelle. Si les frères de SaintGabriel formaient une communauté de taille moyenne, les deux communautés d'origine française dont nous ferons ici état peuvent être qualifiées de très petites, comptant chacune moins de 50 membres en $1964 .^{2}$ Leur contribution éducative n'en fut pas moins marquante au Mont Saint-Bruno.

\section{LES PÈRES TRINITAIRES ${ }^{3}$}

\section{Un ordre très ancien voué aux œuvres de miséricorde}

L'Ordre de la Très-Sainte-Trinité auquel appartiennent les pères Trinitaires de SaintBruno-de-Montarville, compte parmi les plus anciens des grands ordres religieux du monde chrétien. Il fut en effet fondé au $12^{\mathrm{e}}$ siècle, en 1194 plus précisément, à Cerfroid, ville française située à quelque cent kilomètres de Paris. Entourés de quelques disciples, deux jeunes prêtres, Jean de Matha et Félix de Valois, décidèrent de se consacrer aux œuvres de miséricorde, en particulier au rachat ${ }^{4}$ des esclaves très nombreux à cette époque en raison des pirates maures qui sillonnaient les mers. L’Ordre se donna un double but : l'adoration de la Très-Sainte-Trinité et la propagation de son 
culte ainsi que la rédemption et la réintégration sociale des captifs, de religion chrétienne, tombés aux mains des Musulmans, des Turcs et des Maures. ${ }^{5}$ Aujourd'hui, la mission des Trinitaires est restée fondamentalement la même. Ceux-ci se consacrent toujours à la dévotion envers la Sainte-Trinité et poursuivent l'œuvre de charité et de miséricorde, tant spirituelle que corporelle, en se dévouant notamment auprès des pauvres, des malades et, à titre d'aumônier, des prisonniers. ${ }^{6}$

\section{Venue tardive au Canada et à Saint-Bruno-de-Montarville}

Dès la seconde moitié du XIX siècle, Ignace Bourget, infatigable évêque de Montréal, qui de passage à Rome avait assisté à la canonisation du Trinitaire Michel des Saints, entreprend de faire venir des pères Trinitaires pour son diocèse. Le projet est abandonné, mais trois paroisses fondées au nord de Joliette reçoivent le nom de SaintJean-de-Matha, de Saint-Félix-de-Valois et de Saint-Michel-des-Saints en leur honneur. De fait, ce n'est que bien plus tard, en 1924, qu'arrivent les Trinitaires. À l'instar des autres congrégations religieuses implantées ou fondées au Québec de 1915 à 1939 , la « concentration de la population dans les villes, les problèmes sociaux issus de cette concentration et de l'exode rural [...], la constitution de communautés ethniques " amènent les Trinitaires à s'implanter en milieu urbain où l'Église catholique joue alors un rôle social considérable. ${ }^{7}$ Ils fondent une paroisse à Montréal, la paroisse Saint-Jean-de-Matha, dans le quartier populaire de Ville-Émard.

Puis, les Trinitaires connaissent une lente et modeste expansion qui reste limitée à Montréal et à la région sud du Québec. Une expansion qui les amène à acquérir, en 1940, un vaste terrain de quelques centaines d'acres sur le flanc est du mont SaintBruno. La propriété qui compte alors quelques milliers de pommiers et trois petites maisons leur apparaît conforme aux attentes des supérieurs en quête d'un lieu sain, tranquille et propice aux travaux manuels où les aspirants trinitaires pourraient venir passer les vacances d'été. Dès 1947 , le noviciat de la communauté est établi de façon permanente sur le mont Saint-Bruno. L'établissement prend le nom de Couvent de la Trinité-du-Mont. Les effectifs enseignants et étudiants restent fort modestes. Ainsi, en 1950, la maison ne compte que trois pères, trois novices et six frères. En 1957, le noviciat est déménagé à Granby où les pères prennent également la direction d'une paroisse.

\section{Création du Séminaire de la Très-Sainte-Trinité}

L'interruption de la mission enseignante de la congrégation est brève, car dès 1963 les autorités provinciales de l'Ordre décident que les futurs pères Trinitaires y recevraient désormais la plus grande partie de la formation classique. L'établissement est à nouveau agrandi et la partie où se donne la formation académique prend le nom de Séminaire de la Très-Sainte-Trinité. Des pensionnaires, de jeunes adolescents se destinant à la prêtrise, suivent donc les quatre premières années du cours classique (éléments latins, syntaxe, méthode, versification). À l'époque, le Séminaire est affilié à la Faculté des arts de l'Université de Montréal. 
De 1963 à 1965, le nombre d'élèves oscille autour de 70. Mais, avec l'augmentation considérable de population que connaît Saint-Bruno durant les années $1960{ }^{8}$ la clientèle scolaire s'accroît elle aussi. De 83 en 1966, le nombre d'élèves inscrits au Séminaire passe à plus de 130 en 1968. À partir de 1968, les pères dispensent à leurs élèves le cours secondaire instauré par le ministère de l'Éducation du Québec pour remplacer le cours classique.

\section{De modeste collège classique à école secondaire privée réputée}

Selon le Père Rodrigue Coutu, longtemps supérieur de l'institution, « l'insatisfaction créée par les nombreux problèmes vécus dans les écoles alors dites polyvalentes ", incite un groupe de parents de Saint-Bruno à demander aux pères de mettre sur pied un collège privé destiné à recevoir les garçons et les filles de la municipalité.

À cette époque, le Séminaire ne dispose pas des locaux nécessaires et il est de plus soumis à la " clôture ". ${ }^{10}$ Une entente intervient cependant entre les familles et les frères de Saint-Gabriel, communauté religieuse que nous avons évoquée dans une chronique précédente, ${ }^{11}$ qui accepte de louer aux parents des locaux de leur Collège Mont-Gabriel. Avec l'aide des Frères, les pères organisent des classes. Ainsi, en 1970, le Séminaire devient une école secondaire privée, en ouvrant au Collège des frères deux classes de Secondaire I qui accueillent une soixantaine de garçons et de filles en majorité originaires de la municipalité.

Cette situation ne dure que peu de temps, car on l'a vu, les frères louent leur collège à la Commission scolaire de Chambly dès 1972. Les quatre classes de Secondaire I et II doivent réintégrer le Couvent de la Trinité-du-Mont qui doit être agrandi pour accueillir ces nouveaux élèves. Le Séminaire de la Très-Sainte-Trinité devient pour la première fois, mixte. En partie mixte plutôt, car les autorités n'admettent qu'un tiers de filles comme élèves externes et que des garçons comme pensionnaires. ${ }^{12}$

La bonne renommée du collège s'étendant rapidement, les demandes d'admission affluent : en 1974, 700 écoliers déposent une demande d'admission pour 110 places disponibles. Aussi des ajouts successifs sont-ils faits au bâtiment principal de 1974 à 1985 pour ouvrir de nouvelles classes et aménager des locaux destinés à des fins pédagogiques et récréatives. En 1983, la section pensionnat, pour lequel les candidatures diminuent considérablement, est convertie en salles de cours. Le nombre d'élèves admis atteint 500 en septembre 1980 et 625 en 1985. Le programme est le même que dans le secteur public, mais les dirigeants du Séminaire décident d'y enseigner le latin et d'adopter la voie dite " enrichie ». "Enrichie par la forces des choses » selon le Père Rodrigue Coutu, "puisque la demande est si forte que nous pouvons bien sûr choisir la clientèle la plus douée ".

\section{Le retrait des Pères Trinitaires}

Depuis les débuts, les Pères Trinitaires ont enseigné au Couvent puis au Séminaire. Le nombre d'enseignants religieux a cependant fléchi passant de huit en 1972 à seulement trois en 1986. En revanche, le nombre d'enseignants laïcs a beaucoup 
augmenté. Ceux-ci ne sont que quatre en 1972; en 1987, ils sont 35 à se voir confier les diverses disciplines du programme. Aussi, les pères songent-ils alors à mettre l'institution sous la direction d'une corporation formée de religieux et de laïcs. Il leur en coûte de se retirer du Séminaire de la Très-Sainte-Trinité, car même si l'éducation constitue pour eux une œuvre non traditionnelle, elle leur permet d'agir auprès de la jeunesse. En 2000, les Trinitaires doivent cependant se résigner à céder l'administration de leur institution à une corporation laïque.

\section{LES RELIGIEUSES DES SACRÉS-COEURS}

\section{La plus jeune des congrégations de la montagne, fondée pendant les temps troublés de la Révolution française}

C'est à Poitiers en France, en 1797, pendant les temps troublés de la Révolution française, que prend naissance la Congrégation des Sacrés-Cours de Jésus et de Marie et de l'Adoration Perpétuelle du Très Saint-Sacrement de l'Autel. ${ }^{13}$ Henriette Aymer de la Chevalerie et Pierre Coudrin fondent cette congrégation d'hommes et de femmes dont les pratiques de vie religieuse sont inspirées de la Règle de Saint-Benoît. À l'origine, la congrégation a pour but de pratiquer et de propager la dévotion aux SacrésCours et de réparer par l'Adoration perpétuelle (continue) les injustices faites au Seigneur et à sa mère. ${ }^{14}$ Un vif souci d'apostolat pousse cependant très tôt la branche féminine de la Congrégation à se consacrer à l'éducation des enfants issus de toutes les classes sociales.

De leur maison-mère, installée à Paris depuis 1805 sur la rue de Picpus, ${ }^{15}$ les religieuses rayonnent d'abord uniquement en France. Mais à partir de 1835, elles essaiment aux quatre coins du monde : en premier lieu en Amérique du Sud (Chili, Pérou, Bolivie) puis en Europe (Belgique, Angleterre, Espagne, Hollande) à la fin du XIX ${ }^{\mathrm{e}}$ siècle et même en Afrique. En 1908, elles prennent pied pour la première fois en Amérique du Nord en fondant une école à Fairhaven, au Massachusetts.

\section{Leur arrivée en terre canadienne, puis au Québec}

Venues de France, elles débarquent quelque vingt ans plus tard en terre canadienne, en Saskatchewan. Le curé du petit village de Rosetown, l'abbé Emile Gadbois, leur avait demandé de venir y ouvrir une école catholique française. La terrible et longue sécheresse qui sévit dans les Prairies au début des années 1930 associée à une forte crise économique les forcent cependant à quitter la région peu après leur arrivée.

Elles viennent alors s'installer au Québec, aidées en cela par les religieuses de la Congrégation de Notre-Dame. L’Église détient encore dans la province le monopole de l'éducation et les implantations de nouvelles communautés y sont encore très nombreuses. ${ }^{16}$ Toutefois, regroupant moins de 50 membres, les sœurs des SacrésCœurs font partie, à l'instar des Trinitaires, des " plus petites " congrégations religieuses québécoises. Elles ne sont en effet que 28 membres recensées en 1960. ${ }^{17}$ 
Comme bon nombre de communautés religieuses féminines et masculines, les religieuses des Sacrés-Cours se fixent dans la région montréalaise. Elles s'établissent dans un ancien manoir seigneurial situé à Chambly, une petite ville de la région montérégienne, située à quelque 25 kilomètres de Montréal et de Saint-Bruno-deMontarville. À ses débuts, l'établissement qu'elles appellent Val-Marie sert de noviciat et de colonie de vacances. Mais bientôt, les religieuses ouvrent un jardin d'enfants puis une petite école de paroisse et un modeste pensionnat pour garçons et filles de 5 à 12 ans.

\section{À la recherche d'un environnement calme et sain}

En 1953, un incendie détruit la maison Val-Marie de fond en comble. Dans le bâtiment de fortune qu'elles reconstruisent elles-mêmes sur un terrain exigu, les religieuses ne peuvent espérer ouvrir une grande école. De plus, un autre pensionnat est déjà établi dans la région depuis de nombreuses années. Aussi, les religieuses des Sacrés-Cours décident-elles de quitter Chambly. Elles arrêtent leur choix sur SaintBruno-de-Montarville, sur un terrain situé sur le versant est de la montagne, un site dont les grands espaces, le calme et l'air pur leur paraissent convenir à un pensionnat pour jeunes enfants.

Leurs ressources sont toutefois modestes, la maison de Chambly ayant été étant à l'époque le seul établissement d'importance des religieuses des Sacrés-Cours au Québec, ${ }^{18}$ un établissement dépendant de la lointaine province de France. Financièrement soutenues par leurs consœurs du Massachusetts et d'Hawaï, elles peuvent cependant entreprendre en octobre 1954 les travaux de construction d'un pensionnat, un édifice spacieux comprenant aussi un noviciat (voir photo 1) entouré d'un vaste terrain et s'y installent en septembre 1955.

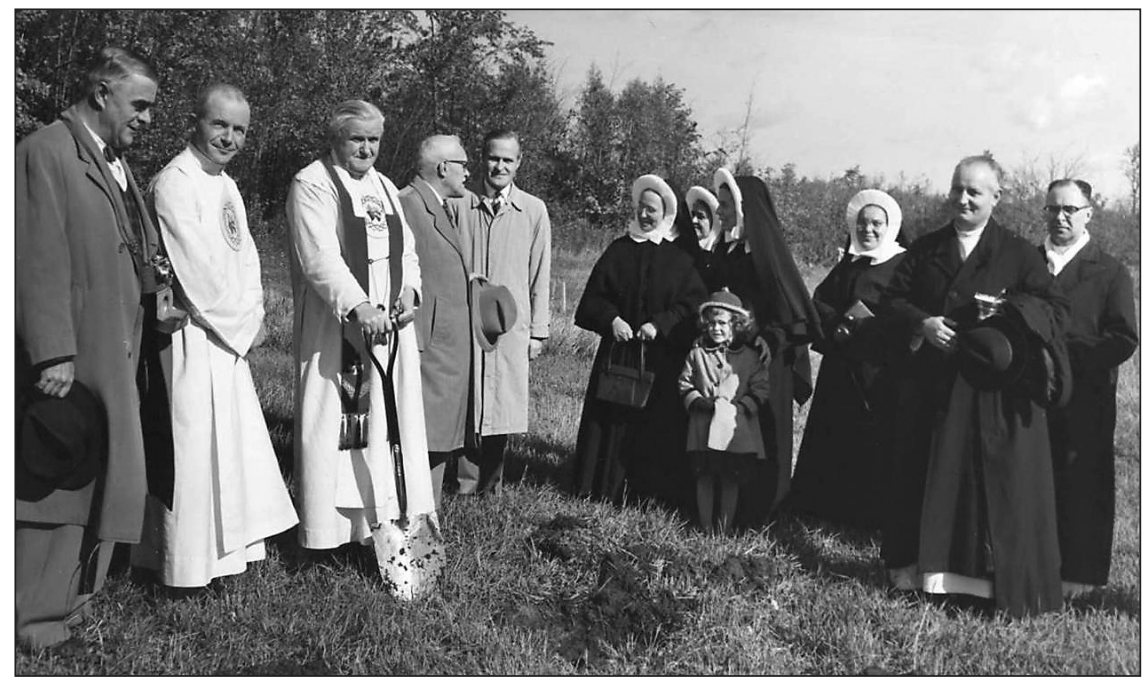

Photo 1 : Première pelletée de terre de la construction du Pensionnat c.1954. Fonds Pensionnat des Sacrés-Coeurs. 


\section{Le Pensionnat des Sacrés-Cœurs : une école primaire en croissance}

C'est à l'automne 1955, une semaine après l'arrivée des religieuses à Saint-Bruno, qu'a lieu la rentrée des enfants au Pensionnat des Sacrés-Cœurs. Il s'agit d'une rentrée modeste avec un total de 68 élèves inscrits de la $1^{\text {re }}$ à la $6^{\mathrm{e}}$ année et répartis en cinq classes. Mais dès l'année suivante, 127 élèves fréquentent les six classes du primaire, comme le montre le tableau 1. Des données tirées des registres du pensionnat permettent d'apprécier le rôle éducatif de cette maison d'enseignement.

\section{Tableau I}

Évolution du nombre de classes, d'élèves, de filles et de garçons au Pensionnat des Sacrés-Cœurs

\begin{tabular}{|c|c|c|c|c|}
\hline Année & $\begin{array}{c}\text { Nombre } \\
\text { des classes }\end{array}$ & $\begin{array}{c}\text { Total des } \\
\text { élèves }\end{array}$ & Filles & Garçons \\
\hline 1955-1956 & 5 & 68 & & \\
\hline 1956-1957 & 6 & 127 & & \\
\hline 1960-1961 & nd & 150 & & \\
\hline 1964-1965 & nd & 185 & & \\
\hline 1969-1970 & 7 & 185 & & \\
\hline 1970-1971 & 7 & 209 & & \\
\hline 1971-1972 & 6 & 207 & & \\
\hline 1972-1973 & 7 & 209 & & \\
\hline 1973-1974 & 7 & 213 & & \\
\hline 1974-1975 & 7 & 209 & 99 & 110 \\
\hline 1975-1976 & 9 & 247 & & \\
\hline 1976-1977 & 8 & 227 & & \\
\hline 1978-1979 & 8 & 228 & & \\
\hline 1979-1980 & 8 & 226 & & \\
\hline 1980-1981 & 8 & 233 & & \\
\hline 1981-1982 & 10 & 287 & & \\
\hline 1982-1983 & 10 & 296 & 152 & 144 \\
\hline 1983-1984 & 12 & 358 & 204 & 154 \\
\hline 1984-1985 & $12^{*}$ & $355^{*}$ & 199 & 156 \\
\hline 1985-1986 & $20^{\circ}$ & $524^{\circ}$ & 259 & 265 \\
\hline 1986-1987 & $20^{\circ}$ & $516^{\circ}$ & 244 & 272 \\
\hline
\end{tabular}

* Sans compter les classes ou les élèves de maternelle

${ }^{\circ} \mathrm{Y}$ compris les classes ou les élèves de maternelle

Sources : Cahiers d'admission, Registres des élèves et Tableaux des inscriptions de 1985-1986 et 1986-1987, Pensionnat des Sacrés-Cours.

À ses débuts, le Pensionnat est une école privée de niveau primaire dispensant un programme accéléré qui permet aux élèves d'accéder au cours classique après six années d'études élémentaires. Après la disparition du cours classique au Québec en 1968, 
l'institution se donne pour but de préparer les enfants au cours secondaire que ces derniers suivent, pour la plupart, dans des établissements privés de la région.

L'année 1969 marque un tournant dans l'histoire du pensionnat. Répondant aux critères gouvernementaux concernant la pédagogie, l'administration et les règlements, le Pensionnat des Sacrés-Cœurs devint la première institution privée à l'élémentaire à recevoir la déclaration d'intérêt public et la subvention gouvernementale qui y est rattachée. Cette subvention s'avère bénéfique pour la maison, car le nombre d'élèves (voir tableau 1) est porté l'année suivante à plus de 200. Au cours de la décennie 1970, le nombre d'élèves et de classes s'accroît légèrement en même temps qu'augmente la population montarvilloise. Cette croissance se poursuit au cours des années 1980 et, malgré la relative stagnation de la population montarvilloise, elle s'accélère même à partir de 1983 en raison notamment de l'ajout d'une classe de maternelle. C'est alors la seule classe de maternelle de Saint-Bruno qui accueille les jeunes enfants durant toute la journée.

Contrairement à ce qui se passe dans le secteur public de l'éducation où l'on répond à la demande, les religieuses suscitent, elles, la demande par l'ajout de classes. C'est que l'institution privée qu'elles dirigent doit atteindre un certain nombre d'élèves pour survivre. Ainsi, c'est pour assurer la viabilité de leur pensionnat que les religieuses décident en 1985 de réaménager complètement leur école et de porter le nombre de classes à 20 pouvant accueillir quelque 525 enfants. ${ }^{19}$

\section{Un rapport pensionnaires/externes, garçons/filles en évolution}

À ses débuts à Saint-Bruno, la maison des religieuses méritait entièrement son nom de Pensionnat puisque tous les élèves, à l'exception d'un seul, étaient pensionnaires. À l'aube des années 1970 toutefois, seulement un peu plus de la moitié des élèves vivent ce régime. Ce nombre encore élevé de pensionnaires contribue d'ailleurs, on l'a vu plus haut, à la reconnaissance de la maison comme institution d'enseignement d'intérêt public. En 1986-1987, le nombre de pensionnaires s'élève à 117, mais ce nombre ne représente plus que $23 \%$ des inscriptions et les pensionnaires passent la fin de semaine dans leur famille. Depuis 2010, la maison d'enseignement ne reçoit plus que des externes qui dînent obligatoirement à l'école en raison du coût élevé du transport scolaire et des besoins des parents.

Au Québec, les sœurs des Sacrés-Cœurs ont toujours accueilli les garçons comme les filles dans leurs maisons. (voir photos 2, 3 et 4) Elles maintiennent cette tradition sans, semble-t-il, faire de discrimination à l'égard de l'un ou l'autre sexe. Les données concernant la proportion de garçons et de filles admis au pensionnat sont incomplètes. Le tableau 1 révèle toutefois que les filles sont plus nombreuses que les garçons de 1982 à 1985. Deux ans plus tard la situation siest toutefois renversée à l'avantage des garçons. Est-ce une tendance passagère, un phénomène dû au hasard? Il est possible que non car certains parents sont persuadés que la fréquentation du Pensionnat des Sacrés-Cœurs constitue une garantie d'admission au réputé Collège des Trinitaires qui n`admet, même pour les garçons, qu>un nombre limité d'élèves. 


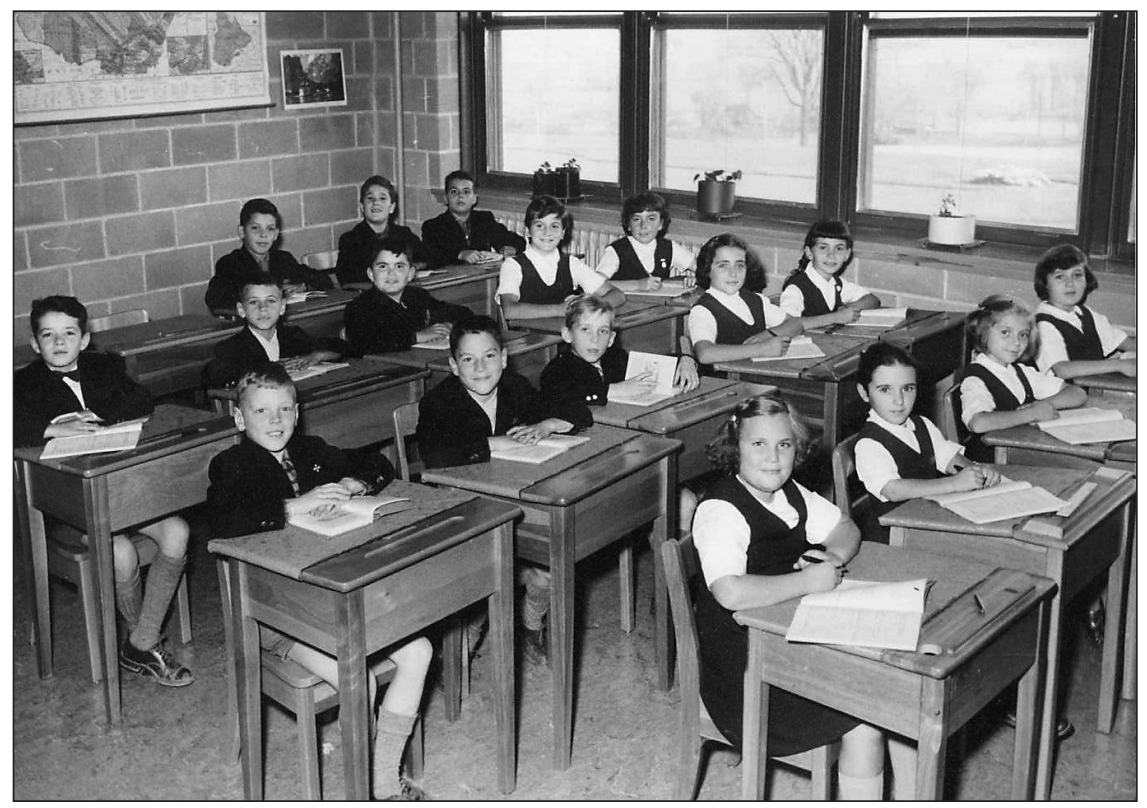

Photo 2 : Classe d'élèves c.1959. Fonds Pensionnat des Sacrés-Cours.

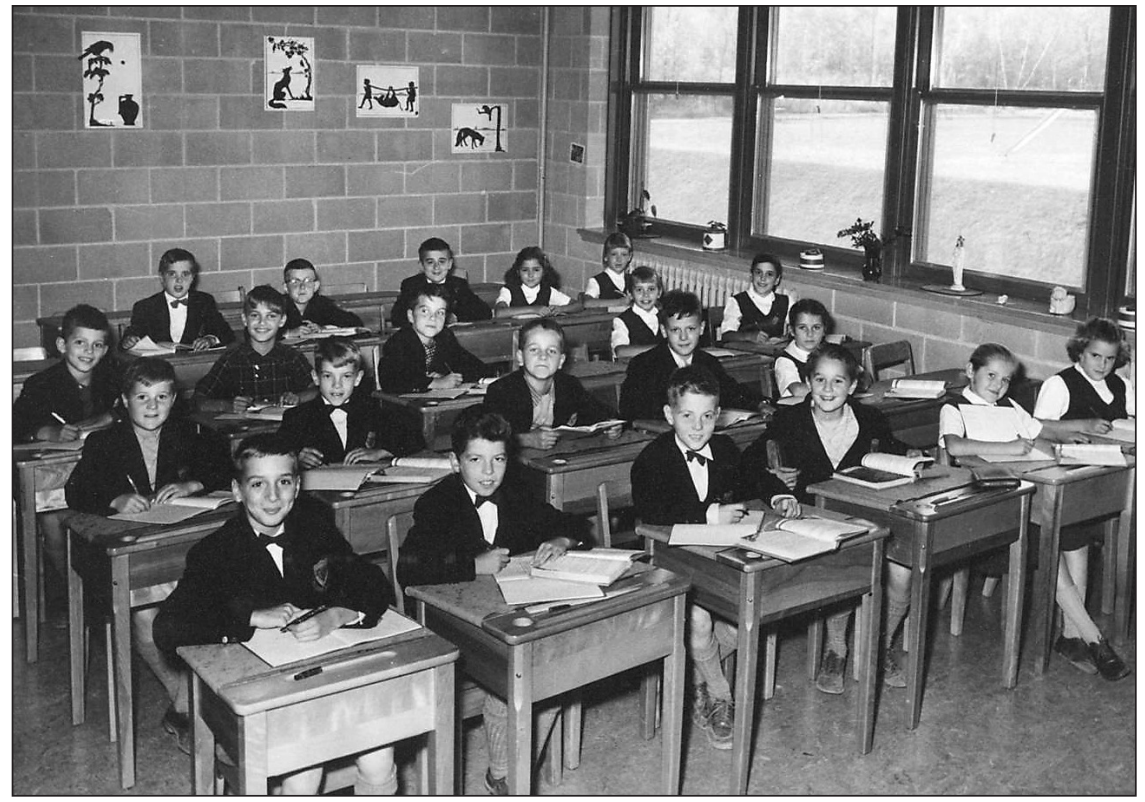

Photo 3: Classe d'élèves c. 1959. Fonds Pensionnat des Sacrés-Coeurs. 


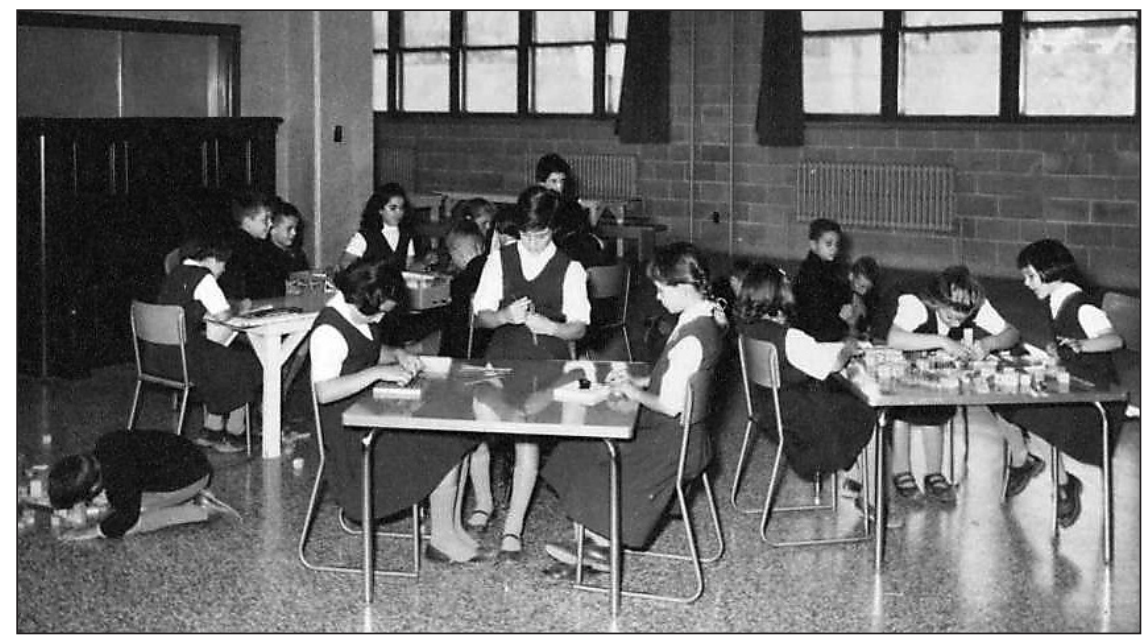

Photo 4 : Travaux pratiques, c.1959. Fonds Pensionnat des Sacrés-Cours.

\section{Un recrutement de clientèle de plus en plus local}

Durant les premières années d'existence de l'établissement, bon nombre d'élèves fréquentant l'établissement, pensionnaires pour la plupart, viennent de Montréal. C'est que le Pensionnat est la seule institution de niveau primaire du district de Montréal à recevoir des pensionnaires. ${ }^{20}$

Mais, déjà à la fin de la décennie 1960, les jeunes Montréalais ne constituent plus qu'une minorité. Ils ont été remplacés par des enfants venant des diverses municipalités de la Rive-Sud et par de jeunes Montarvillois qui constituent une proportion sans cesse croissante de la clientèle scolaire. Si bien qu'en 1974-1975, 69 des 209 élèves de l'école, soit le tiers des effectifs étudiants, résident à Saint-Bruno. Dix ans plus tard, les jeunes Montarvillois et Montarvilloises forment la communauté la mieux représentée parmi les 355 élèves du primaire, même si le Pensionnat attire un nombre croissant d'élèves originaires de Sainte-Julie, de Saint-Basile, de Beloeil et d'autres municipalités des environs. Quant aux jeunes Montréalais, ils ne constituant plus que quelque $5 \%$ de la clientèle. Au tournant des années 1980, c'est essentiellement à Saint-Bruno et dans la région immédiate que le Pensionnat des Sacrés-Cœurs recrute désormais sa clientèle.

\section{Des religieuses enseignantes en nombre décroissant}

Le nombre total de religieuses se maintient à 32 jusqu'au milieu des années 1960 puis décline à 26 en 1968 et à 16 en 1973 (voir photo 5). En 1980, il ne reste que sept religieuses demeurant au Pensionnat. Ce petit nombre permet cependant d'augmenter le nombre de classes et d'élèves du pensionnat sans qu'il y ait besoin pendant longtemps d'agrandir la maison. De fait, le bâtiment n'est agrandi qu'une fois, en 


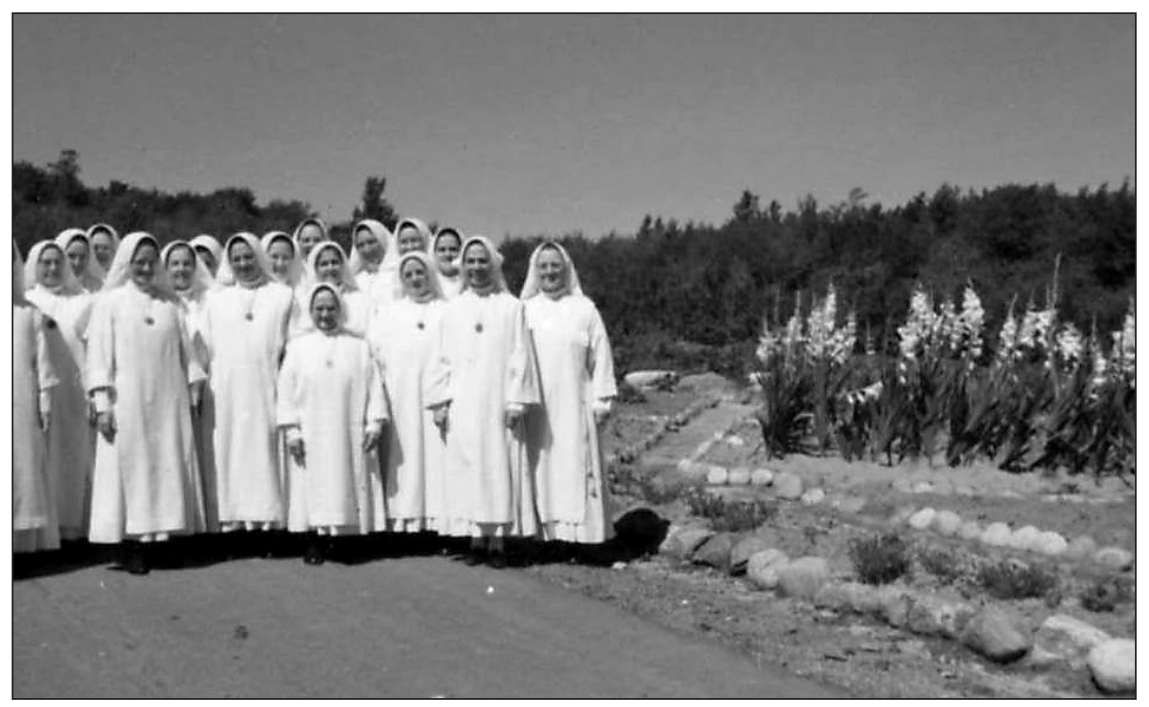

Photo 5 : Soeurs dans le jardin entourant le Pensionnat, c 1970. Fonds Pensionnat des Sacrés-Coeurs.

1985 (voir photo 6), et ce, par l’occupation des derniers locaux encore occupés par les religieuses.

Pour maintenir et développer leur établissement, les religieuses ont donc dû faire appel à des laïcs. Une première institutrice laïque est engagée en 1959. Durant les décennies 1960 et 1970, quelques enseignants laïcs s'ajoutent aux religieuses dont

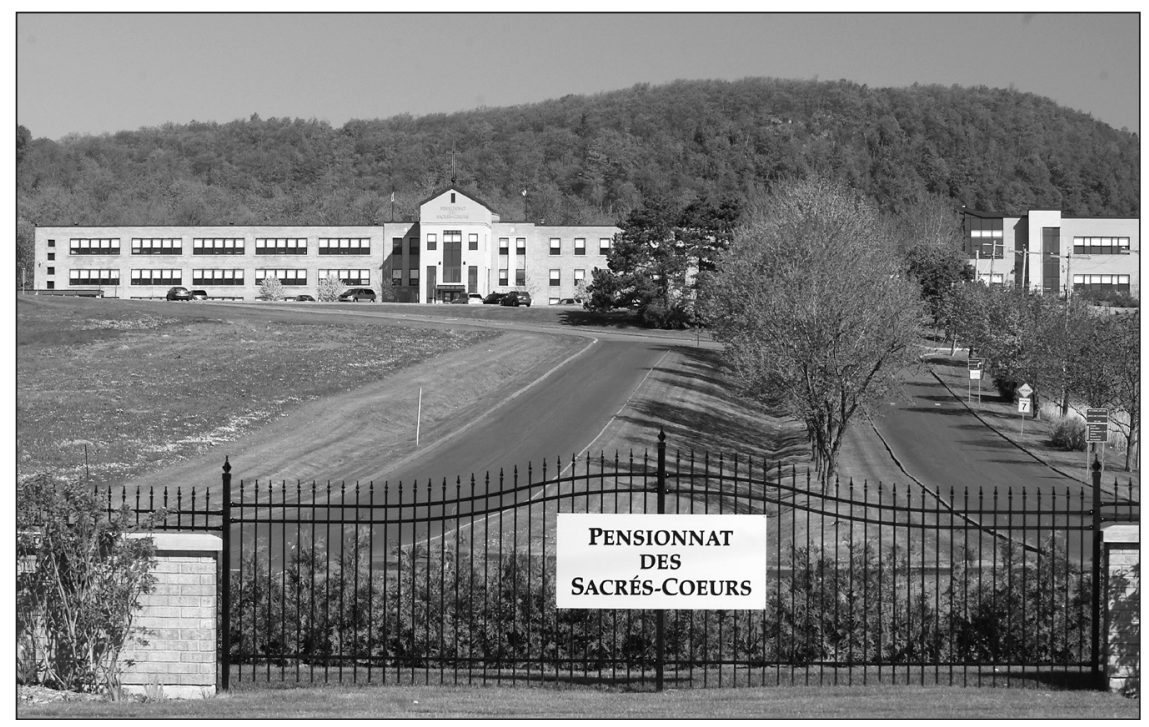

Photo 6: Le Pensionnat des Sacrés-Coeurs aujourd'hui. Fonds Pensionnat des Sacrés-Cours. 
le nombre reste supérieur à ceux-ci. A partir des années 1980 cependant, le nombre d'enseignants laïcs, hommes et femmes, dépasse le nombre de religieuses. Et la tendance à la laïcisation du corps professoral s'accentue fortement à la suite du décès de quatre religieuses et au départ pour l'étranger et surtout pour des paroisses voisines de la plupart des membres de la Congrégation. ${ }^{21}$ En 1987, il ne reste plus que trois religieuses enseignantes alors que les laïcs sont une cinquantaine. Aussi n'est-il pas étonnant qu'en 1998, les sœurs des Sacrés-Cœurs décident de laisser la direction de leur établissement à une corporation laïque et transferent leur école à un groupe d'administrateurs bénévoles qui assurent la pérennité de la mission éducative de l'établissement. En effet, quelque 800 enfants fréquentent aujourd'hui cette école primaire des plus réputées.

\section{En guise de conclusion}

Les trois congrégations religieuses qui firent œuvre éducative au Mont Saint-Bruno étaient d'origine européenne, française précisément. Les frères de Saint-Gabriel puis les Trinitaires et les religieuses des Sacrés-Cœurs sont venus au Canada pour répondre à des besoins précis, pour remplir des tâches d'enseignement et de charité que la société canadienne-française confiait traditionnellement à l'Église catholique.

Aucune des trois congrégations ne s'est établie directement au Mont-Saint-Bruno. C'est l'existence en flanc de montagne de vastes espaces à prix accessible et exploitables et encore en retrait du développement résidentiel qui les a vraisemblablement attirées.

Profitant davantage de l'explosion démographique de la municipalité, les derniers arrivés au Mont-Saint-Bruno, les Trinitaires et les religieuses des Sacrés-Cœurs, ont rempli une mission éducative importante. Si ces deux toutes petites communautés religieuses ont dû abandonner la formation de leurs propres sujets, elles ont pu pendant de longues années, aidées de nombreux enseignants laïcs, dispenser dans des institutions privées, un enseignement réputé, notamment auprès de la population montarvilloise.

En raison de leur arrivée tardive au Québec et de l'existence de nombreuses communautés religieuses enseignantes bien établies dans la province, les Trinitaires et les religieuses des Sacrés-Cœurs n'ont pu cependant exercer une œuvre éducative importante hors de Saint-Bruno. S'ajoute à cela le fait que l'effectif total des congrégations religieuses au Québec atteint son sommet dès 1961 en raison de la diminution des nouvelles vocations. Puis s'amorce un déclin attribuable à la diminution de l'influence de l'Église et à la prise en main par l'État du champ de l'éducation. ${ }^{22}$ Déjà de très petites congrégations, les religieuses des Sacrés-Cœurs et les Trinitaires doivent céder la direction de leurs établissements à des corporations laïques au tournant des années 1990. Auparavant, aux prises eux aussi avec un déclin de leurs effectifs, les frères de Saint-Gabriel avaient dû quitter le collège qu'ils avaient mis sur pied en 1925 et se replier sur l'île de Montréal en 1978.

Il n'en demeure pas moins que le souvenir de l'œuvre éducative de ces trois congrégations enseignantes demeure encore bien vivace au sein de la population montarvilloise. 


\section{Notes}

1 Voir Andrée Dufour, «Trois congrégations religieuses enseignantes au Mont SaintBruno : 1. Les Frères de Saint-Gabriel ", Historical Studies in Education/Revue d'histoire de l'éducation, vol. 23, $\mathrm{n}^{\circ} 2$ (automne 2011) : 129-134.

2 Bernard Denault et Benoît Lévesque, Éléments pour une sociologie des communautés religieuses au Québec (Sherbrooke et Montréal : Les Presses de l'Université de Montréal, 1975), 55, 198-199.

3 Les études portant sur l'œuvre des Trinitaires au Québec sont fort rares. Nous nous sommes appuyée ici sur deux courts textes écrits : Maryse Demers, "Les Pères Trinitaires " paru dans La petite histoire de la Paroisse St-Bruno, s.l., 1981-1982, Lise Dumont "L'œuvre des Pères Trinitaires à Saint-Bruno, Cahiers de la Société d'histoire de Beloeil et de Mont Saint-Hilaire, n 40 (1993), p. 33-40 et Carole Clément, " Le Père Rodrigue, né pour l'enseignement ", Le Journal de Saint-Bruno, mardi le 10 juin 1986, p. 12. Soulignons cependant que des entrevues du Père Rodrigue Coutu, longtemps directeur de l'institution, faites le 12 février 1985 et le 12 mars 1987 et du Frère Damien Mercier, faites le 30 mars et le 14 avril 1987 nous ont apporté des renseignements précieux.

4 Il s'agissait de l'achat de l'esclave au moyen d'une rançon.

5 Sur cet ordre religieux, on peut lire Roseline Grimaldi-Hierholz, L'Ordre des Trinitaires : histoire et spiritualité (Paris : A. Fayard, 1995), 238 p.

6 À propos de cette mission au Québec, mentionnons le refuge «La Maison du Père " où les Trinitaires se consacrent depuis 1970 à l'accueil et à la réinsertion sociale des hommes itinérants.

7 B. Denault et B. Lévesque, Éléments..., 92-95.

8 De 5700 personnes en 1960, la population montarvilloise passe à 14911 personnes en 1970.

9 De 1968 à 2001, on désignait ainsi les écoles secondaires qui offraient une formation générale et une formation technique qui permettaient l'obtention d'un diplôme d'études secondaires (DES). Aujourd'hui, la formation technique ayant à toutes fins pratiques disparue de ces établissements, on les désigne simplement sous le nom d'écoles secondaires.

10 Ce qui signifie que le Séminaire était "fermé », interdit aux laïcs, aux femmes en particulier.

11 Voir la note 1.

12 Selon le Père Rodrigue Coutu, les filles avaient davantage de collèges privés à leur disposition dans la région. De plus, selon lui, il y avait plus d'abandons du côté des filles en raison des sciences.

13 Au Québec, la communauté porte le nom, heureusement abrégé, de Congrégation des Sacrés-Cœurs et de l'Adoration perpétuelle. Pour simplifier, les termes de religieuses, de sœurs ou de Congrégation des Sacrés-Cœurs seront employés dans ce texte.

14 Père Fleury-Dives. La Congrégation des Religieuses des Sacrés-Cours et de l'Adoration perpétuelle (Picpus). Coll. «Les Ordres religieux » (Paris : Letouzy et Ané, 1924), 129-140.

15 De là, le nom de Sours de Picpus avec lequel on désigne familièrement, et gentiment, les Sœurs des Sacrés-Cœurs.

16 Soixante-quatre communautés religieuses s'installèrent au Québec entre 1943 et 1965 (B. Denault et B. Lévesque. Éléments..., 96).

17 B. Denault et B. Lévesque, Éléments ..., 55. Ces auteurs estiment que les « très grandes » communautés d'hommes et de femmes au Québec comptent plus de 1000 membres. Sept communautés religieuses de femmes ont compté plus de 1000 membres, et cinq plus de 2000 membres. 
18 En 1967, elles acceptent cependant la direction de deux écoles paroissiales, l'une de garçons, l'autre de filles, au village de Lac-à-la-Croix (au Lac Saint-Jean) où les pères des Sacrés-Cœurs dirigent un séminaire ("Les Sœurs des Sacrés-Cœurs au Canada » dans Horizons blancs. "Spécial Canada ", n 24, juillet 1965, 206-208).

19 Selon Sœur Lise Chartrand lors de sa présence à la réunion de la Société d'histoire de Montarville du 8 avril 1986.

20 "Les Sœurs des Sacrés-Cœurs au Canada »..., 208.

21 En 1987, seulement 3 des 22 religieuses des Sacrés-Cœurs encore présentes au Québec enseignent. Les autres religieuses sont à Beloeil (alors lieu de résidence principal de la congrégation), à Saint-Hubert, à Saint-Constant et à Ville Lemoyne. Les religieuses de Saint-Hubert et de Saint-Constant vivent l'expérience d'insertion en paroisse, car depuis le Concile de Vatican II, depuis 1975 surtout, les religieuses se sont également donné pour mission de témoigner de leur foi par leur présence effective, en vivant, auprès de gens plus défavorisés.

22 B. Denault et B. Lévesque, Éléments ..., 96-102. 